\title{
BARKHAUSEN NOISE EMISSION IN MILLED SURFACES
}

This paper deals with the non-destructive evaluation of surface made of roll bearing steel 100Cr6 after milling cycles via Barkhausen noise technique. The paper discusses magnetic anisotropy linked with the structure transformations with regard to variable flank wear of cutting tool. Effective value of Barkhausen noise (BN), Peak Position and Full Width at Half Maximum (FWHM) derived from the raw BN signals are compared with metallographic observations and residual stress profiles. Surface integrity is also expressed in term of surface roughness due to comparable BN sensitive layer and the height of surface roughness profile.

Keywords: Barkhausen noise, milling, surface integrity.

\section{Introduction}

Cyclic magnetization in a ferromagnetic material produces magnetic pulsation as a result of irreversible and discontinuous Bloch Walls (BW) motion. Discontinuous BW motion is due to interference of BW with microstructure features such as precipitates, dislocation cells, grain boundaries and other lattice defects [1 - 4]. This phenomenon is named Barkhausen noise [5]. Barkhausen noise (BN) technique has found high industrial relevance mainly for monitoring surfaces loaded near their physical limits. BN features are usually correlated with residual stresses, microhardness or structure transformations. This technique is mostly adopted for monitoring of surfaces after grinding cycles due to strong correlation among thermal over tempering, associated surface burn and the corresponding BN features [6 and 7]. Effect of thermal softening after grinding due to over tempering decreases dislocation density (and the corresponding surface hardness), transforms carbides shape and their morphology as well as produces tensile stresses [6 and 7]. All the above mentioned aspects contribute to the higher magnitude of BN. Being so, surface over tempering can be easily revealed by the use of the Barkhausen noise technique [7].

Nowadays, it can be found that BN technique has been employed for the next industrial applications. BN technique was successfully adapted for turning operations [ 8 and 9]. These studies indicate strong influence of flank wear $V B$ on surface integrity expressed in such BN features as BN (represent its effective rms value), Peak Position or BN envelope profile. As it was found, remarkable decrease of $\mathrm{BN}$ values and the simultaneous increase of Peak Position along with the progressively developed $V B$ are linked with increasing dislocation density and the corresponding structure transformations [ 8 and 9]. Moreover, verification of such concept in the real industrial conditions also revealed strong correlation between surface integrity after turning cycles (expressed in BN values) and deformations (expressed in their ovality and roundness deviation) produced by the consecutive heat treatment (hardening). Being so, this paper reports about the similar problem of milling operation in which $\mathrm{BN}$ emission, metallographic observations and residual stress profiles are investigated on surfaces after milling cycles produced by inserts of variable $V B$. Aspect of surface roughness is also involved due to comparable BN sensitive layer and the height of surface roughness profile.

\section{Experimental conditions}

Experiments were conducted on samples made of annealed bearing steel $100 \mathrm{Cr} 6.10$ pieces of dimension $60 \times 43 \times 25 \mathrm{~mm}$ were prepared for long term test. Cutting process was monitored as a long term test where such aspects as flank wear $V B$, structure alterations and corresponding surface integrity expressed in magnetoelastic responses (BN) of the milled surface were investigated. Cutting and other conditions: milling machine - FA4 $\mathrm{AV}$, dry cutting, cutting tool made of cemented carbides R3001240E-PM, R300-050Q22 - 12M 262489 of diameter $\emptyset 50 \mathrm{~mm}$ with 2 inserts of variable flank wear $V B$ (in the range from 0.05 to $0.8 \mathrm{~mm}), a_{p}=0.25 \mathrm{~mm}, v_{f}=112 \mathrm{~mm} \cdot \mathrm{min}^{-1}, v_{c}=78.5 \mathrm{~m} \cdot \mathrm{min}^{-1}$. Flank wear was measured for both cutting inserts and $V B$ values indicated in the paper represent their average value.

\footnotetext{
* Miroslav Neslusan, Tomas Hrabovsky, Maria Cillikova, Anna Micietova

Department of Machining and Manufacturing, Faculty of Mechanical Engineering, University of Zilina, Slovakia

E-mail: miroslav.neslusan@fstroj.uniza.sk
} 

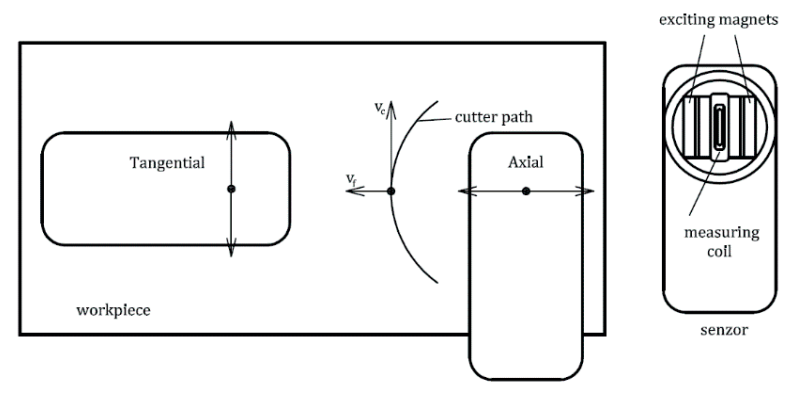

Fig. 1 Orientation of $B N$ sensor

BN measurement was performed by the use of RollScan 300 and software package $\mu$ Scan in the frequency range of 10 to 1000 $\mathrm{kHz}$ (magnetizing frequency $125 \mathrm{~Hz}$, magnetizing voltage $4 \mathrm{~V}$ ). Each BN value was determined by averaging of 10 consecutive BN bursts (5 magnetizing cycles). Due to strong surface anisotropy, each surface was measured in two directions - tangential and axial as Fig. 1 illustrates. BN values indicated in the paper represent the effective (rms) value of BN signal. To reveal the microstructure transformations induced by milling $10 \mathrm{~mm}$ long pieces were sectioned and routinely prepared for metallographic observations (etched by $2 \%$ Nital for $8 \mathrm{~s}$ ). Microstructure was observed in the direction of cutting speed. Residual stresses were measured by mechanical method based on electrolytic etching of ( 2 hours, $20 \%$ concentration of $\mathrm{H}_{2} \mathrm{SO}_{4}$ - electrolyte, $5 \mathrm{~V}$ and $6 \mathrm{~A}$ ) machined surface and simultaneous measurement of a sample deformation. The details about principle, mathematic apparatus and measuring unit can be found in [10]. Surface roughness was measured by the use of Hommel Tester T 2000 in direction of feed speed (axial direction, see Fig. 1).

\section{Results of experiments}

Figure 2 depicts two different phases of flank wear VB. Turning operations are not usually performed with the inserts of flank wear VB above $0.5 \mathrm{~mm}$ in order to avoid the excessive cutting forces and catastrophic tool failures. On the other hand, the flank wear is a major factor affecting the thickness of heat affected zone (HAZ), see Figs. 3, 4, 5 and 6. For this reason, quite large $\mathrm{VB}$ were employed to facilitate surface of relative thick HAZ; thus making more remarkable specific aspect of surface integrity investigated via BN. Microstructure observations show that milling with inserts of low VB $(0.05$ and $0.2 \mathrm{~mm})$ produces surface containing thin thermally softened layer which appears dark in the near surface region (see Fig. 3). HAZ is not continuous of variable thickness below $2 \mu \mathrm{m}$. As soon as the VB becomes more developed thickness of HAZ progressively increases. The higher VB is employed, the thicker HAZ can be produced (see Figs. 5 and 6).

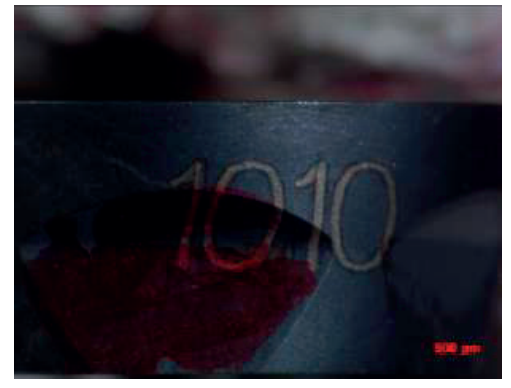

a) sharp cutting insert, $V B=0.05 \mathrm{~mm}$

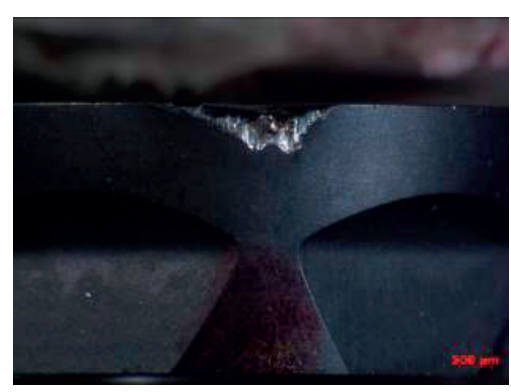

b) $V B=0.8 \mathrm{~mm}$

Fig. 2 Two different phases of flank wear VB

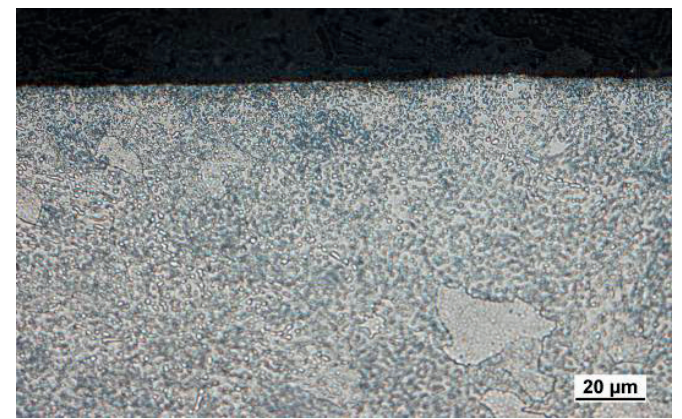

Fig. 3 Microstructure of milled surface, $V B=0.05 \mathrm{~mm}, \mathrm{HAZ}(2 \mu \mathrm{m})$

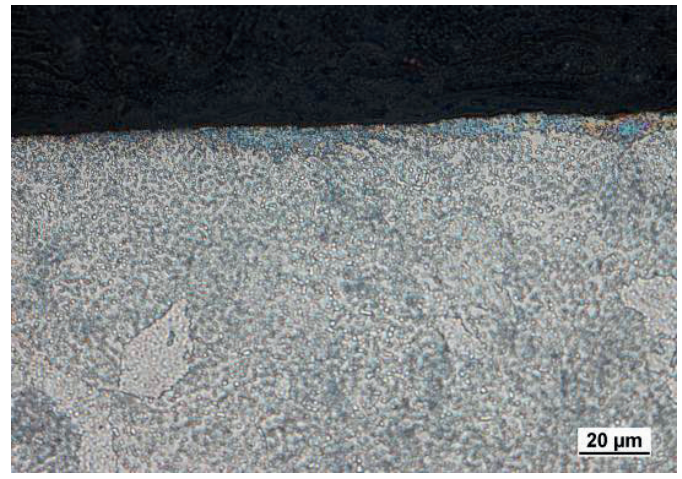

Fig. 4 Microstructure of milled surface, $V B=0.4 \mathrm{~mm}, \operatorname{HAZ}(3.6 \mu \mathrm{m})$ 




Fig. 5 Microstructure of milled surface, $V B=0.6 \mathrm{~mm}, \mathrm{HAZ}(5 \mathrm{um})$

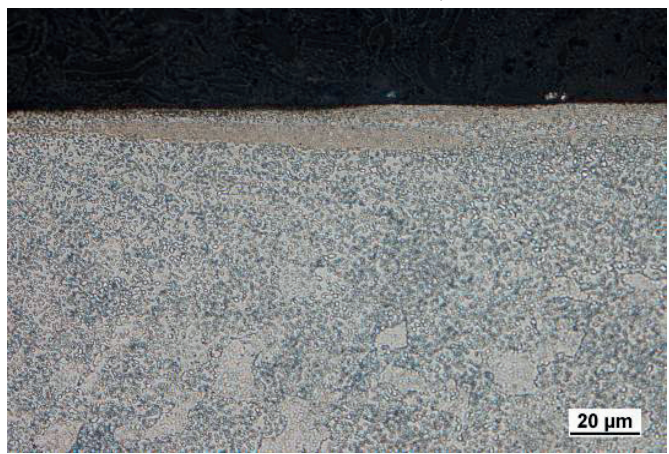

Fig. 6 Microstructure of milled surface, $V B=0.8 \mathrm{~mm}, \mathrm{HAZ}(5.5 \mu \mathrm{m})$

Figures 7, 8 and 9 depict influence of VB on surface roughness either illustrated by the raw roughness profiles (see Fig. 7) or via the extracted parameters (see Figs. 8 and 9). These figures reveal transformations in cutting edge geometry as well as intensification of plastic deformation of the machined surface. Due to a certain cutting edge radius a thin layer defined by the stagnation point undergoes severe plastic deformation during the time period corresponding the flank wear land. Both these aspects contribute to the progressive increase of surface roughness expressed in variable parameters. Furthermore, as the surface roughness profile increases in its height, the height of its irregularities

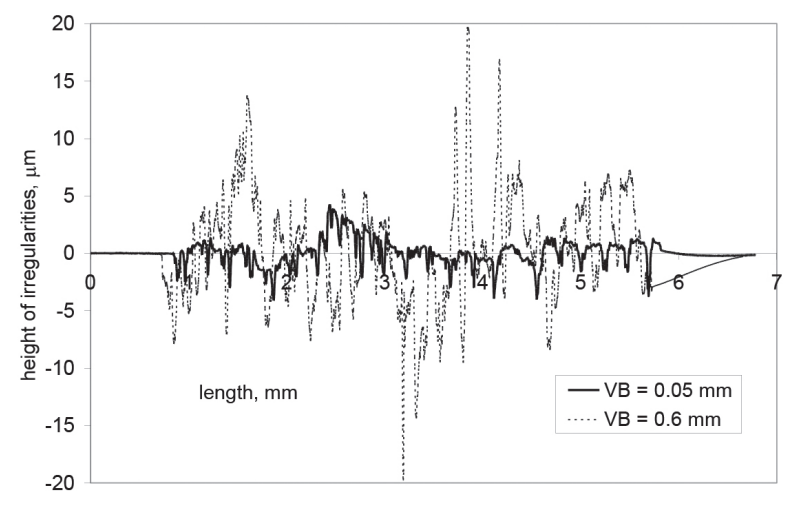

Fig. 7 Surface roughness profiles becomes comparable with $\mathrm{BN}$ sensitive layer (the estimated BN sensitive thickness is about $40 \mu \mathrm{m}$ ). Being so, the volume of the structure within the surface roughness height (expressed in $\mathrm{Rz}$ parameter) contributing to the $\mathrm{BN}$ signal received on the free surface is reduced as the VB becomes more developed. It is considered that this effect contributes to the lower magnitude of BN obtained for surfaces produced by inserts of higher VB.

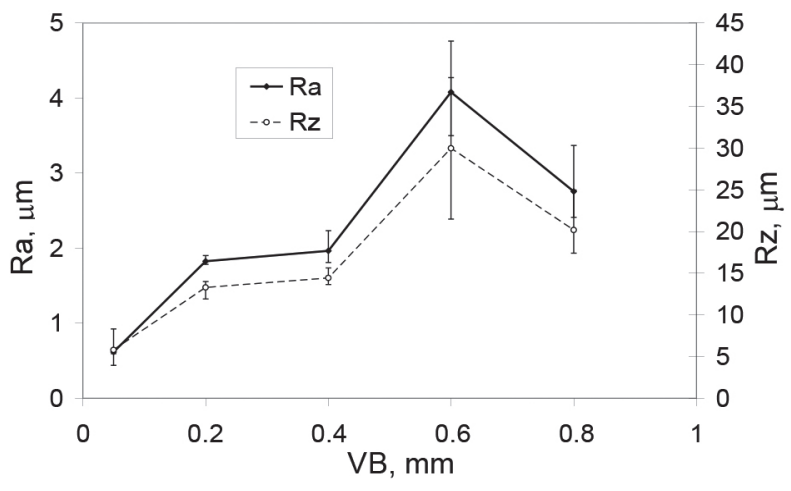

Fig. $8 R a$ and $R z$ versus $V B$

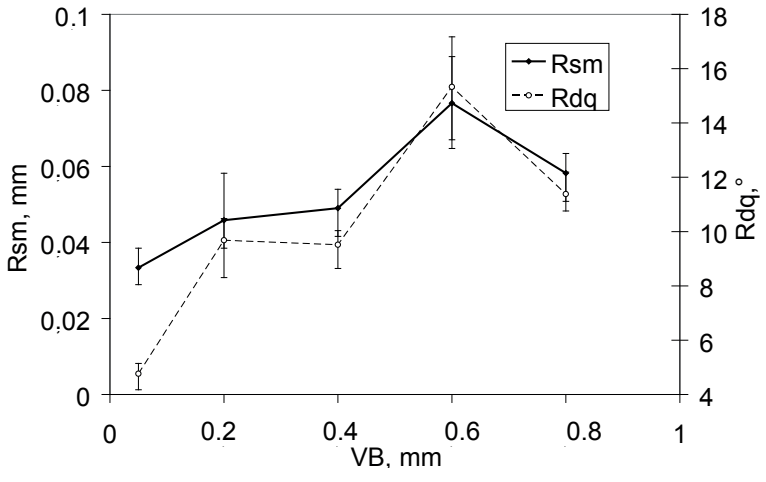

Fig. 9 Rsm and Rdq versus VB

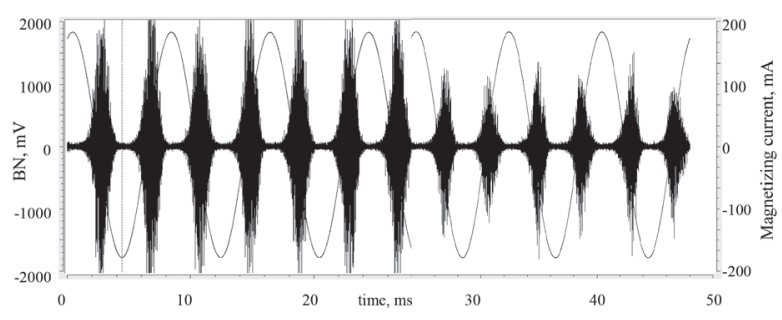

a) $V B=0.2 \mathrm{~mm}$

b) $V B=0.6 \mathrm{~mm}$

Fig. 10 BN signals in tangential direction

It is worth to mention that the generic term HAZ can not be associated with the pure thermal load (and corresponding thermal softening) of the near surface region. Due to a certain cutting edge radius and the corresponding stagnation point 
a certain layer ahead of a cutting edge is not separated from the sample as a part of the chip but undergoes the cutting edge and forms the near surface region of machined surface exposed to the severe plastic deformation at elevated temperatures. It is well known that machined surface after turning or milling cycle usually contains near surface region in which effect of plastic deformation dominates whereas the deeper layers are mainly thermally softened. Both these (usually contradictory) effects (strain hardening and thermal softening) affect microstructure of machined surface; thus BW motion and $\mathrm{BN}$ emission. Both layers, near surface strain hardened and deeper thermally softened affects BN emission after milling cycle in a synergistic manner.

Figure 10 illustrates the $\mathrm{BN}$ signals obtained for tangential direction at two different VB. This figure shows that surface produced by inserts of lower $\mathrm{VB}$ emits $\mathrm{BN}$ signals of higher magnitude than that produced by the insert of higher VB. Figure 10 shows evolution of $\mathrm{BN}$ in the different directions. Figure 10 indicates richer $\mathrm{BN}$ emission in tangential direction (associated with the direction of cutting speed) than that in the axial direction (associated with the direction of feed speed). Strong magnetic anisotropy is due to unbalance between cutting and feed speeds as a result of stress anisotropy and associated effect of magnetostriction [11].

Figure 11 shows that BN drops down along with the progressive developed VB. Progressive decrease of BN versus VB in both directions is linked with the structure transformations in the near surface layer as a region mostly contributing to the BN signal obtained on the free surface. Gradual decrease of BN values in both directions indicates that effect of surface hardening dominates over the effect of thermal softening. Such behavior was previously explained (turned surfaces) through interference of BW with structure containing higher dislocations density [8] due to remarkable strain hardening at more developed VB. Higher dislocation density at higher VB is connected with two aspects as follows:

- transformations in cutting edge geometry (increasing cutting edge radius, more negative rake angle, etc.),

- longer time interval during which machined surface undergoes severe plastic deformation corresponding to the flank wear land.

As it was reported [8 and 12], both aspects affect $\mathrm{BN}$ in a synergistic manner. On the other hand, neither evolution of Peak Position nor FWHM indicate any remarkable variation versus VB (except Peak Position for VB $=0.8 \mathrm{~mm}$ ), see Fig. 12 . Also residual stress profiles (see Fig. 13) indicate mainly tensile stresses. As VB becomes more developed the higher magnitude of tensile stresses can be obtained. It is well known that tensile stresses are a product of thermal effect (unbalance in heating and cooling of neighboring layers - I type stresses). It is well known that tensile stresses contribute to the richer $\mathrm{BN}$ emission whereas compressive stresses reduce BN magnitude. Being so, it is apparent that microstructure features (pinning sites in the structure interfering with BW) take the major role in $\mathrm{BN}$ emission whereas stress state is only minor.

It is also worth to mention that thermal softening in machining cycles becomes more significant in the case of hardened samples whereas surface strain hardening becomes more visible on metallographic observations (visible texture or refined white layer) when more negative tool geometry (rake angle) is employed. Moreover, such tool geometry also contributes to the remarkable increase of Peak Positions and transformation of $\mathrm{BN}$ envelopes profiles [8 and 12]. Nevertheless, $\mathrm{BN}$ emission in this study exhibits continuous drop down along with $\mathrm{VB}$ and

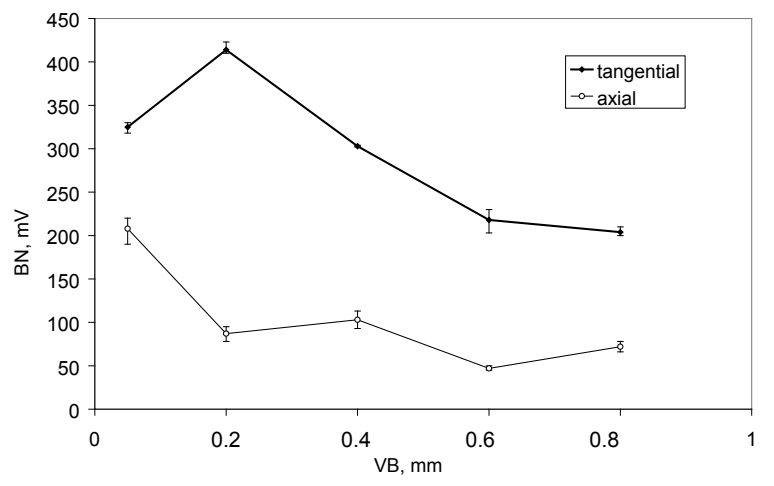

Fig. 11 BN versus VB

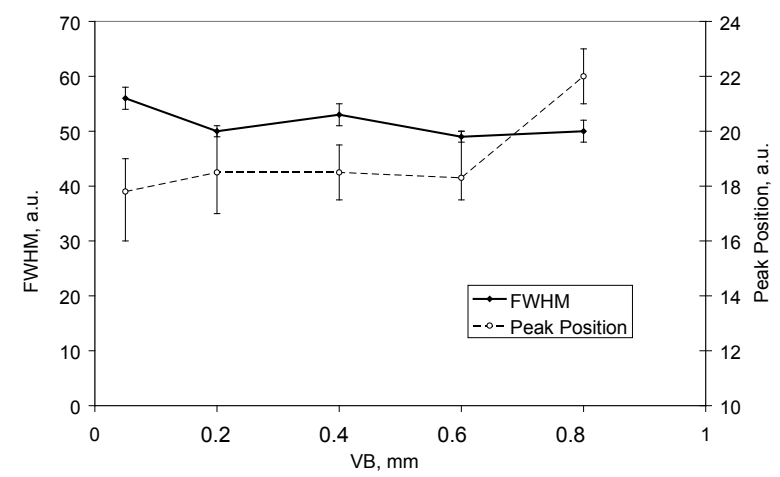

Fig. 12 Peak Position and FWHM versus VB

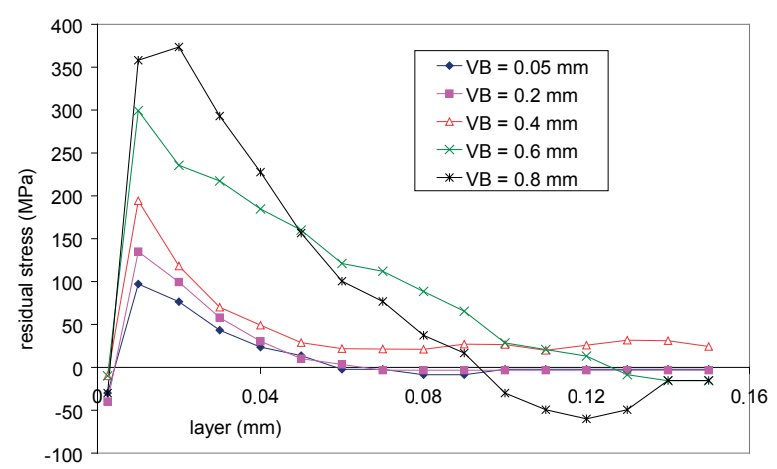

Fig. 13 Residual stress profiles 
can be easily employed for non destructive monitoring of surface integrity after milling. On the other hand, further experiments focused on microhardness profile and measurement of dislocation density should be carried out to verify the above mentioned information.

\section{Conclusions}

BN technique has found a high industrial relevance for detection mainly grinding cycles when unacceptable thermal softening and tensile stresses contribute to the high $\mathrm{BN}$ values. Milling or turning processes form the different state of surface integrity than that after grinding. Grinding cycles usually produce isotropic (from magnetic as well as stress point of view) surface whereas turning and milling cycles do not. However, this study demonstrates and proves the potential of $\mathrm{BN}$ technique for monitoring surfaces of high anisotropy. Industrial implementation of BN technique for real needs would avoid implementation of components of unacceptable surface; thus prevent their early crack initiation and machine premature failures.

\section{Acknowledgement}

This article was edited under the financial support of KEGA projects No. 005ZU - 4/2014, 009ZU - 4/2014.

\section{References}

[1] KAMEDA, J., RANJAN, R.: Nondestructive Evaluation of Steels using Acoustic and Magnetic Barkhausen Signals - II. Effect of Intergranular Impurity Segregation, Acta Metall., vol. 35/7 1987, pp. 1527-1531.

[2] BUTTlE, D. J., SCRUBY, C. B., JAKUBOVICS, J. P., BRIGGS, G. A. D.: Magneto-acoustic and Barkhausen Emission: Their Dependence on Dislocations in Iron, NDT \& E Int., vol. 24 1991, pp.47-54.

[3] GATELIER-ROTHEA, C., et al.: Characterization of Pure Iron and Carbon-iron Binary Alloy by Barkhausen Noise Measurements: Study of the Influence of Stress and Microstructure, Acta Mater., vol. 46/14, 1998, 4873-4882.

[4] RANJAN, R., JILES, D.C., RASTOGI, P.: Magnetic Properties of Decarburized Steels: An Investigation of the Effects of Grain Size and Carbon Content, IEEE Trans. Magn., vol. 23/3, 1987, 869-1876.

[5] BARKHAUSEN, H.: Phys. Zeitschrift, vol. 20, 1919, pp. 201.

[6] MICUCH, M., CILliKOVA, M., NESLUSAN, M., MICIETOVA, A.: Micromagnetic Study of Cutting Conditions and Grinding Wheel Wear Influence on Surface Integrity, Manufacturing Technology, vol. 14 2014, 66-71.

[7] ROSIPAL, M.: Application of Barkhausen Noise for Study of Surface Integrity of Machined Surfaces, PhD. Dissertation, University of Zilina, 2012.

[8] CiZEK, J., NESLUSAN, M. CilliKoVA, M., MiCIETOVA, A. MELIKOVA, O.: Modification of Steel Surfaces Induced by Turning: Non-destructive Characterization using Barkhausen Noise and Positron Annihilation, J. of Physics D: Applied Physics, vol. 47, 2014, 1-17.

[9] DUBEC, J., NELUSAN, M., FAKTOR, M.: Analysing the Influence of Tool Wear when Turning, on Bearing Rings Deformation through Barkhausen Noise, Technolog, vol. 1, 2013, 16-21.

[10] NECKAR, F.: Influence of Cutting Process on Surface Integrity, Research report No. 400, CVUT : Praha, 1985.

[11] HAJKO, V., POTOCKY, L., ZENTKO, A.: Magnetization Processes, ALFA : Bratislava, 1982.

[12] DUBEC, J., NESLUSAN, M.: Multiparametric Analysis of Surface after Turning through Barkhausen Noise in Relation to Tool Wear, MM Science, July 2012. 